\title{
Pole Position
}

Dear Reader,

The German Federal Minister of Transport Andreas Scheuer has made a bold announcement: "Germany will be the international number 1 in autonomous driving. We will be the first country in the world to bring autonomous vehicles from research laboratories onto roads: and used in daily operation. (...)." The next step has now been taken with the autonomous driving law that creates a legal framework to enable autonomous vehicles (level 4) [editor's note: probably referring to automated vehicles] to operate regularly in defined operating areas on public roads. This regular operation could therefore start in 2022.

The automotive industry will ensure the implementation and they will need to intensify their (already considerable) efforts in the domain of automated driving. Two competing approaches can be currently observed: a more evolutionary path via the continuous extension of available assistance systems, and a more revolutionary path via robotic vehicle fleets with safety drivers, in order to completely leapfrog the intermediate levels SAE $2+$ to 4 as fast as possible. The latter makes sense, since then there are no legacy issues; however, it is a huge leap. There is also an associated risk as surveys show that at the moment, a high percentage of people say they would not trust an autonomously driving vehicle.

The situation describing how we approach SAE level 5 and whether evolution or revolution is a step ahead remains tense and exciting. For example, Tesla as a representative of evolution, published announcements in social media at the end of 2020 concerning a new version of its Full Selfdriving: According to Elon Musk, the system is close to SAE level 5.
However, what is interesting to read in this context is that Waymo CEO John Krafcik, as a proponent of the revolutionary approach, is convinced that Tesla and thus actually all others - will never be able to reach SAE level 5 using an evolutionary approach. Despite the fact that many share this view, not least because Tesla uses comparatively low-cost sensors, the thorny issue remains whether a policy of small steps can really result in an autonomous system.

Whatever the score is, the German legal framework is not enough on its own.

The goal must be to unify the legal situation for automated driving and its validation on a European level. A European legal framework is important: At the end of the day, whoever is number 1 in automated driving, they would have fewer hurdles to take if they don't have to follow too many different national regulations.

Enjoy reading this edition!

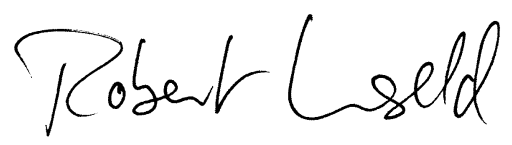

Robert Unseld Responsible Editor

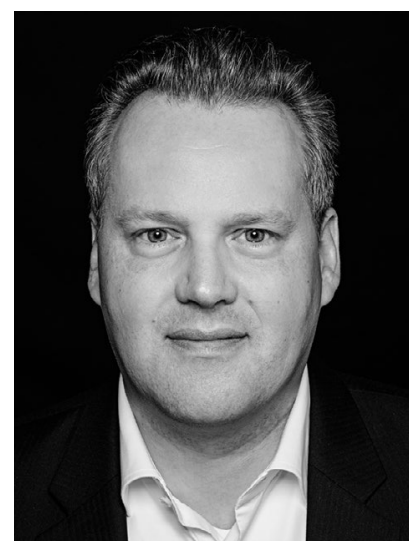

\section{PCAN-MiniDiag FD}

Compact handheld for basic diagnosis of CAN and CAN FD buses. Measurement of bit rate, termination, bus load, and voltages at the D-Sub connector.

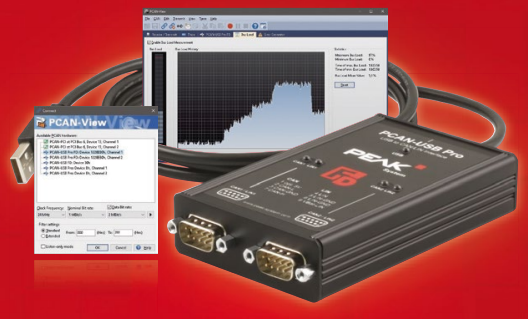

\section{PCAN-USB Pro FD}

High-speed USB 2.0 interface for the connection of 2 CAN FD and 2 LIN networks with galvanic isolation.

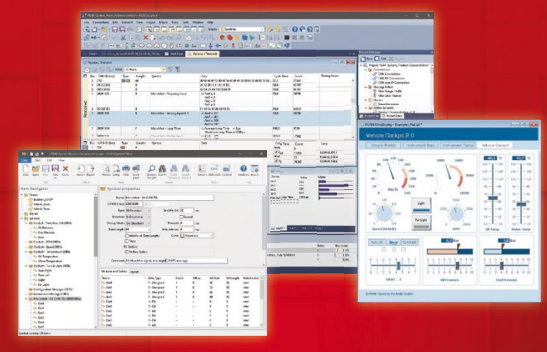

\section{PCAN-Explorer 6}

Professional Windows software for observation, control, and simulation of CAN FD and CAN 2.0 buses. 